\title{
Gesundheitsversorgung am Lebensende variiert nach Region
}

\author{
PhD Nicole Steck, PhD Claudia Berlin, Prof. Marcel Zwahlen
}

Institut für Sozial- und Präventivmedizin (ISPM) in Bern

Folgende Forschende des ISPM haben an den hier vorgestellten Projekten mitgearbeitet: Professor Kerri Clough-Gorr, Professor Matthias Egger, Professor Marcel Zwahlen, Xhyljeta Luta, PhD, Maud Maessen, PhD, und Radoslaw Panczak, PhD.

\begin{abstract}
Angesichts der Alterung der Schweizer Bevölkerung nimmt die Bedeutung der Gesundheitsversorgung am Lebensende zu. Viele Fragen sind aber noch offen. Forschende des Instituts für Sozial- und Präventivmedizin (ISPM)* in Bern haben unter anderem regionale Unterschiede bei der Intensität der Spitalpflege, wo die Leute sterben sowie bei den Kosten am Lebensende untersucht.
\end{abstract}

Die End-of-Life-Care, das heisst die medizinische und pflegerische Versorgung am Lebensende, ist in den vergangenen Jahren zunehmend in den Fokus gerückt. So haben Bund und Kantone im Jahr 2009 die Nationale Strategie Palliative Care beschlossen und in den vergangenen Jahren umgesetzt. Mit Lebensende sind die letzten Tage, Wochen oder Monate vor dem Tod gemeint. Damit kann End-of-Life zwar jede Altersgruppe betreffen, aber über $80 \%$ der in der Schweiz versterbenden Personen sind älter als 65 Jahre. Der Schweizerische Nationalfonds (SNF) hat im Jahr 2012 das Schwerpunktprogramm NFP 67 «Lebensende» gestartet [1]. Dieses soll dazu beitragen, dass diese Lebensphase und Themen wie Patientenverfügung, Zugang zu Palliative Care, Suizidhilfe sowie Betreuung sterbender Angehörigen besser verstanden werden. Der im November

\section{Zusammenfassung}

Angesichts der zunehmenden Alterung der Schweizer Bevölkerung nimmt die Bedeutung der Gesundheitsversorgung am Lebensende zu. Mit Lebensende sind die letzten Tage, Wochen oder Monate vor dem Tod gemeint. Der Schweizerische Nationalfonds (SNF) hat im Jahr 2012 das Schwerpunktprogramm NFP 67 «Lebensende» gestartet. Dieses soll dazu beitragen, dass Themen wie Patientenverfügung, Zugang zu Palliative Care, Suizidhilfe sowie Betreuung sterbender Angehörigen besser verstanden werden. Der vor drei Jahren verstorbene Versorgungsforscher Professor André Busato hat innerhalb des NFP 67 am Institut für Sozial- und Präventivmedizin (ISPM) in Bern verschiedene Projekte initiiert. Die nun vorliegenden Resultate dieser Studien zeigen, dass in der Schweiz am Lebensende bezüglich der Intensität der Gesundheitsversorgung, des Orts des Sterbens und der Kosten grosse regionale Unterschiede bestehen. Während die Sprachregion und zum Teil die bestehende Infrastruktur einige der Unterschiede erklären können, konnte für die untersuchten sozioökonomische Faktoren der Patienten kein Zusammenhang gezeigt werden. Die Unterschiede sind zum Teil gerechtfertigt, etwa durch unterschiedliche Erkrankungen und Präferenzen der Patienten. Ein Teil der Varianz dürfte aber auch ungerechtfertigt und damit unerwünscht sein. Um die genauen Hintergründe zu verstehen und mögliche Gegenmassnahmen zu identifizieren, sind weitere Untersuchungen nötig.
2013 verstorbene Versorgungsforscher Professor André Busato hat innerhalb des NFP 67 am ISPM in Bern verschiedene Projekte initiiert, die nun zu einem grossen Teil abgeschlossen sind. Deren Ergebnisse werden hier vorgestellt.

\section{In der Westschweiz sind die Menschen am Lebensende länger im Spital}

Die Untersuchung der Intensität der Gesundheitsversorgung am Lebensende birgt Fragen: Welche Zeitspanne vor dem Tod wird untersucht? Wie wird Intensität definiert und quantifiziert? Das End-of-Life-Team des ISPM hat in einer systematischen Übersichtsarbeit untersucht, wie solche Fragen in der Literatur angegangen wurden [2]. Aus über 1500 Artikeln wurden 58 Studien identifiziert, die die Intensität der Gesundheitsversorgung am Lebensende untersucht und quantifiziert hatten. Die untersuchte Zeitspanne variierte von 48 Stunden bis zu zwölf Monaten vor dem Tod. Zur Beurteilung der Intensität der Gesundheitsversorgung am Lebensende wurden von den meisten Studien Hospitalisationen, Einweisungen auf die Intensivstation sowie Chemotherapien verwendet. Diese Indikatoren wurden jedoch kaum validiert, und eine breit abgestützte Expertenmeinung fehlt.

Die Forschenden nahmen sich anschliessend der Intensität der Gesundheitsversorgung am Lebensende in der Schweiz an. Insbesondere ging es ihnen um regionale Unterschiede bei Patienten, die im Spital verstarben. Sie verwendeten dazu Daten der medizinischen Statistik der Krankenhäuser (s. Kästchenmeldung). Patienten wurden in die Studie eingeschlossen, wenn sie über 18 Jahre alt waren und zwischen Spitaleinweisung und Tod maximal sechs Monate lagen. Insgesamt wur- 
den die Daten von 24400 im Jahr 2010 Verstorbenen analysiert [3]. In ihren letzten sechs Lebensmonaten wurden diese durchschnittlich 2,2-mal ins Spital eingewiesen und verbrachten insgesamt knapp einen Monat (29 Tage) in einem Krankenhaus. Fast jeder Dritte wurde mindestens einmal auf die Intensivstation verlegt, wo er im Mittel 39,7 Stunden verbrachte. Eine multivariable Regressionsanalyse zeigte unter anderem, dass Krebspatienten länger im Spital blieben als Menschen, die nach Unfällen, Herz-Kreislauf-Erkrankungen, Infektionen oder Erkrankungen des Verdauungstrakts oder der Lunge eingeliefert wurden. Ganz junge (19- bis 40-jährige) und ganz alte Menschen (über 90) waren in ihren letzten sechs Lebensmonaten im Schnitt weniger lang im Spital. Zwischen den Sprachregionen zeigten sich deutliche Unterschiede: In der Westschweiz waren die End-of-Life-Patienten im Durchschnitt länger im Spital als in der Deutschschweiz. Eine Ausnahme bildete unter anderem die Deutschschweizer Stadt Basel, wo ältere Menschen um einen Fünftel länger im Spital blieben als im nationalen Durchschnitt. Allgemein zeigten sich bei älteren Menschen grössere regionale Unterschiede als bei jüngeren.

\section{Regionale Unterschiede auch beim Sterben in Institutionen}

Die meisten Menschen möchten zu Hause sterben [4]. Eine Auswertung des Schweizerischen Gesundheitsobservatoriums Obsan ergab jedoch, dass in der Schweiz in den Jahren 2006 bis 2011 über drei Viertel aller Menschen in einem Spital oder in einem Pflegeheim gestorben sind [5]. Das End-of-Life-Team des ISPM hat ein Rahmenkonzept erarbeitet, das die Determinanten für Unterschiede in der medizinischen Versorgung am Lebensende in drei Kategorien einteilt [6]: Auf der Macroebene spielen generelle Umstände wie

\section{Dialoggruppe Forschungsschwerpunkt Versorgungsforschung}

Versorgungsforschung ist für die Ärzteschaft ein wichtiger und wegweisender Wissenschaftsbereich. In Zeiten des Umbruchs und der Veränderungen im Gesundheitswesen (neue Finanzierungsmodelle, demographische Veränderungen, steigende Gesundheitskosten usw.) ist eine akademisch verankerte Forschung im Bereich der ärztlichen Versorgung zwingend nötig. Dies hat auch der Nationalfonds erkannt und 2015 mit dem NFP 74 «Smarter Health Care» ein nationales Schwerpunktprogramm zur Versorgungsforschung lanciert (http://www.snf.ch/de/fokusFor schung/nationale-forschungsprogramme/nfp-74/).

Um wissenschaftliche, von Partikulärinteressen unabhängige Grundlagen schaffen zu können, unterstützen die Verbindung der Schweizer Ärztinnen und Ärzte (FMH), die Konferenz der Kantonalen Ärztegesellschaften (KKA) sowie Newlndex gemeinsam den Forschungsschwerpunkt Versorgungsforschung am ISPM Bern. Eine Dialoggruppe dient als Informations- und Austauschplattform: Vertreter der Organisationen und der Forschungsgruppen diskutieren regelmässig die laufenden und geplanten Arbeiten im Bereich der Versorgungsforschung. Die Dialoggruppe verfolgt zudem das Ziel, die Akzeptanz und Sensibilisierung innerhalb der Ärzteschaft für diesen Wissenschaftsbereich zu fördern.

Die Dialoggruppe steht ihrer Basis offen für Themen-, Diskussionsvorschläge sowie für weitere Fragen und Informationen. Die Abteilung Daten, Demographie und Qualität DDO der FMH übernimmt die Koordination der Dialoggruppe und steht für weitere Informationen und Auskünfte gerne zur Verfügung: ddq[at] fmh.ch oderTel. 0313591111.

sozioökonomische und politische Faktoren, die Gesundheitspolitik sowie der kulturelle Hintergrund eine Rolle. Auf der Mesoebene finden sich Eigenschaften und Vorlieben des Gesundheitspersonals sowie das lokale Angebot und die Organisation der Gesundheitsversorgung. $\mathrm{Zu}$ den Determinanten auf der Microebene zählen persönliche Eigenschaften und Vorlieben der Patienten und der Pflegenden, aber auch Art und Schwere der Erkrankung (s. Abb. 1).

Anhand dieser drei Ebenen an Determinanten hat das Team gut 41000 Todesfälle von über 65-jährigen Men-
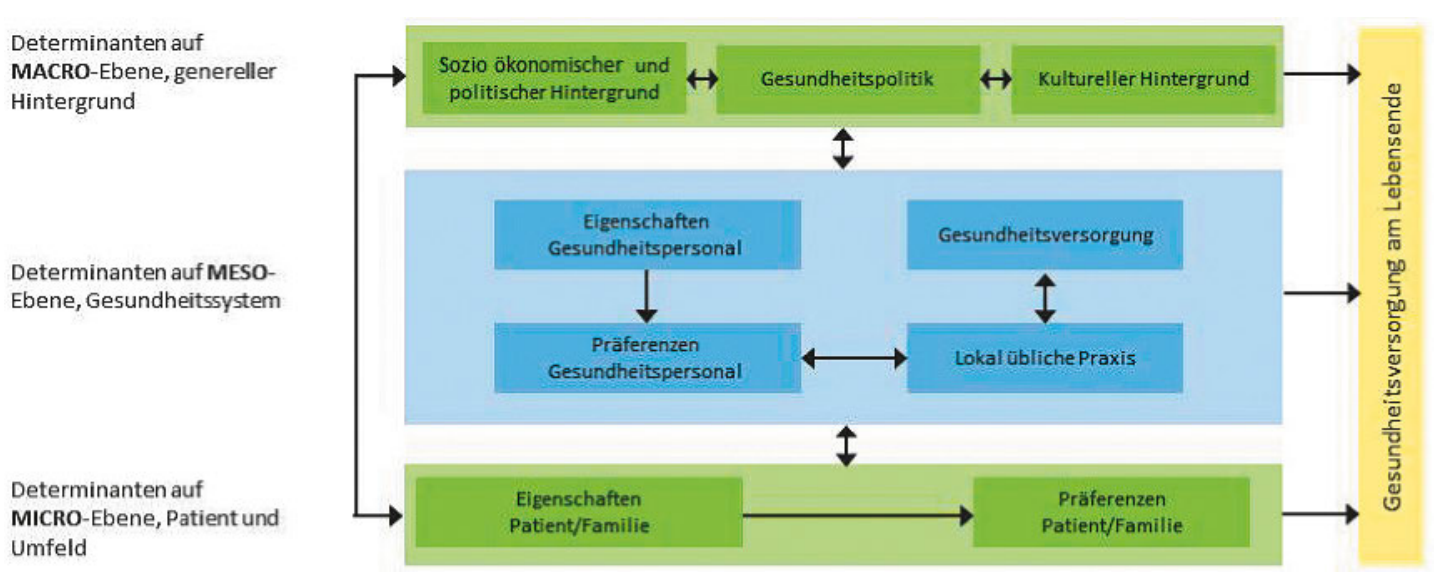

Abbildung 1: Mögliche Determinanten für Unterschiede der Gesundheitsversorgung am Lebensende. 
schen in Institutionen in der Schweiz untersucht und regionale Differenzen analysiert [7]. Dies entspricht rund $80 \%$ aller Todesfälle von über 65 -Jährigen im Jahr 2010. Die Analyse der Todesfälle in Institutionen zeigte, dass der Anteil der Todesfälle in Pflegeheimen mit zunehmendem Alter anstieg und bei den über 90-Jährigen knapp 80\% erreichte. Frauen verstarben deutlich seltener im Spital als Männer. Aber nicht nur individuelle Eigenschaften scheinen zu beeinflussen, wo jemand stirbt: Regional gesehen hatten Menschen aus stadtnahen Gebieten eine höhere Wahrscheinlichkeit, in Spitälern zu sterben als Menschen aus den Städten selber oder vom Land. Zudem starben Menschen in der Romandie und dem Tessin häufiger im Spital als jene in der Deutschschweiz. Eine höhere Dichte an ambulanten Ärzten sowie an ambulanten Pflegeheimen war mit einer geringeren Wahrscheinlichkeit des Sterbens im Spital assoziiert.

\section{Die Gesundheitskosten im letzten Lebens- jahr variieren stark}

Die Gesundheitskosten sind im letzten Lebensjahr durchschnittlich fünfmal höher als in den Jahren davor. Um Unterschiede je nach Region und Dienstleister zu untersuchen, haben die Forscher eine kleinräumige Analyse der Gesundheitskosten im letzten Lebensjahr durchgeführt [8]. Dazu wurden die Abrechnungsdaten von sechs Schweizer Krankenversicherungen (CSS, Groupe Mutuel, Helsana, Sanitas, SWICA, Visana) verwendet, die rund 60\% der 2008-2010 verstorbenen Menschen abdecken. Diese anonymisierten Daten wurden mit der Todesursachenstatistik des Bundesamtes für Statistik (BFS) verknüpft, um fehlende Informationen wie Todesursache, Nationalität, Zivilstatus sowie Religion hinzuzufügen. Die FMH und das BFS stellten zudem weitere Informationen zu den medizinischen Leistungserbringern und Kapazitäten sowie zur Bevölkerung zur Verfügung.

Es wurden alle von den Krankenkassen verarbeiteten Rechnungen der letzten zwölf Monate der 113277 Versicherten analysiert, die zwischen 2008 und 2010 verstarben. Im Durchschnitt betrugen die Ausgaben für medizinische Leistungen im letzten Lebensjahr 32500 Schweizer Franken. Die höchsten Kosten generierten junge Personen, die an Krebs (insbesondere kolorektale, Brust- und Prostatatumore) starben. In der «teuersten" Region waren die Kosten bei der rohen Analyse um den Faktor sieben höher als in der "günstigsten" Region. Diese Unterschiede nahmen ab, wenn verschiedene Faktoren berücksichtigt wurden, insbesondere Alter, Todesursache und Sprachregion (s. Abb. 2). Hingegen hatte das medizinische Versorgungsangebot in der Region keinen Einfluss auf die Kosten. Nicht alle regio-

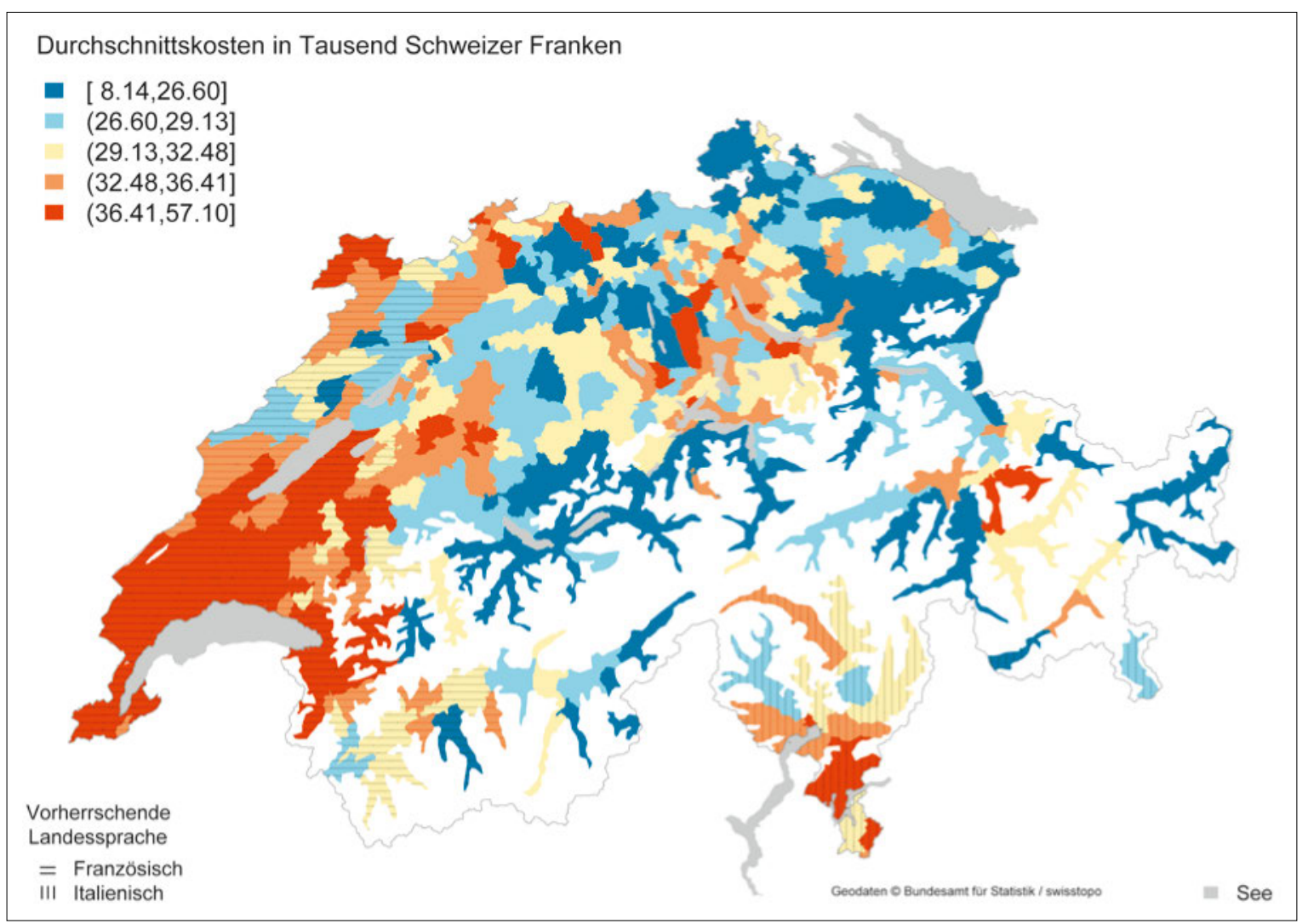

Abbildung 2: Gesundheitskosten in den letzten 12 Lebensmonaten. 
nalen Kostenunterschiede konnten in der Studie erklärt werden. So gab es etwa bei den älteren Frauen auch nach Korrektur aller Faktoren noch beträchtliche Kostenunterschiede im letzten Lebensjahr. Die deutliche Reduktion der Kostenunterschiede beim Einbezug der Sprachregion deutet darauf hin, dass die Organisation der Versorgung am Lebensende in den Sprachregionen und somit auch den kulturellen Regionen der Schweiz unterschiedlich gehandhabt wird.

\section{Suchen Alleinstehende weniger medizi- nische Hilfe im letzten Lebensjahr?}

In einer weiteren Analyse der Krankenversicherungsdaten ging es um die Menschen, für die bei ihrer Krankenkasse keine Rechnung für die letzten zwölf Lebensmonate vorlag. Das End-of-Life-Team untersuchte, inwieweit persönliche Merkmale der Patienten und ihr Wohnort eine Rolle dabei spielten [9].

Vor allem junge, alleinstehende, verwitwete und geschiedene Personen sowie Personen, bei denen eine Erkrankung aus dem Kapitel "psychische und Verhaltensstörungen" (zum Beispiel Demenz) als Todesursache verzeichnet wurde, hatten in den letzten zwölf Monaten vor ihrem Tod keine Abrechnungen. Sie erkennen möglicherweise die Notwendigkeit medizinischer Hilfe weniger, beanspruchen verspätet Hilfe oder sind mit dem administrativen Aufwand überfordert. Allerdings müssen die Gründe, warum keine Abrechnungen vorlagen, in weiteren Studien untersucht werden.

\section{Analysen mit Routinedaten bergen Probleme}

Alle Analysen des End-of-Life-Teams basierten auf Daten, die in früheren Jahren zu statistischen und administrativen Zwecken erhoben wurden. Entsprechend waren die Daten von unterschiedlicher Qualität und nicht zur Beantwortung aller Fragen geeignet. Wichtige Informationen wie der Schweregrad der Erkrankungen, die Präferenzen der Patienten und ihrer Familien sowie die gängige Praxis waren nicht verfügbar. Dennoch zeigten die Studien, dass in der Schweiz am Lebensende grosse regionale Unterschiede bestehen bezüglich der Intensität der Gesundheitsversorgung im Spital, des Orts des Sterbens und der über die Krankenkassen abgerechneten Kosten. Die Sprachregion und zum Teil die bestehende Infrastruktur können einige der Unterschiede erklären, aber bei weitem nicht alle. Ein Teil der Unterschiede ist gerechtfertigt, etwa durch unterschiedliche Krankheiten oder auch

\section{Medizinische Statistik der Kranken- häuser und Krankenhausstatistik}

Wichtige Quellen für die End-of-Life-Forschung am ISPM sind die vom Bundesamt für Statistik (BFS) erhobene medizinische Statistik der Krankenhäuser sowie die Krankenhausstatistik.

Die medizinische Statistik der Krankenhäuser erfasst jedes Jahr die anfallenden Daten aller Hospitalisierungen in den Schweizer Krankenhäusern. Es werden sowohl soziodemographische Informationen der Patienten wie Alter, Geschlecht, Wohnregion als auch administrative Daten wie Versicherungsart oder Aufenthaltsort vor der Hospitalisierung und medizinische Informationen wie Diagnosen und Behandlungen erhoben.

Die Krankenhausstatistik dient in erster Linie zur Beschreibung der Infrastruktur und der Tätigkeit der Spitäler und Geburtshäuser der Schweiz. Die Betriebe erstatten jährlich Bericht unter anderem über die erbrachten ambulanten und stationären Leistungen, das Personal sowie ihre Betriebsrechnung. Die Datenerhebung dient statistischen und administrativen Zwecken.

Präferenzen der Patienten. Ein Teil der Variabilität dürfte aber auch ungerechtfertigt und damit unerwünscht sein. Um die Hintergründe genauer zu verstehen und mögliche Gegenmassnahmen zu identifizieren, sind weitere Untersuchungen nötig.

\section{Bildnachweise}

@ Institut für Sozial- und Präventivmedizin Bern

\section{Literatur}

1 Schweizerischer Nationalfonds. NFP 67 Lebensende. 2012. http:// www.snf.ch/de/fokusForschung/nationaleforschungsprogramme/nfp67-lebensende/Seiten/default.aspx

2 Luta X, Maessen M, Egger M, Stuck AE, Goodman DC, Clough-Gorr KM. Measuring intensity of end of life care: a systematic review. PloS One 2015;10(4):e0123764. doi:10.1371/journal.pone.0123764.

3 Maessen M, Panczak R, Luta X, Stuck AE, Egger M, Goodman DC, Zwahlen M, Clough-Gorr KM. The intensity of care during the last six months of life: an analysis of Swiss administrative hospital data. Manuscript under review.

4 Rainsford S, MacLeod RD, Glasgow NJ. Place of death in rural palliative care: A systematic review. Palliative medicine 2016;30(8): 74563. doi: $10.1177 / 0269216316628779$.

5 Obsan. Auswertung Sterbeort 2006-2011. 2013. http://www.bag. admin.ch/themen/gesundheitspolitik/13764/13777/13793/index. html?lang=de

6 Luta X, Panczak R, Maessen M, Stuck AE, Egger M, Goodman DC, Clough-Gorr KM. Understanding variation in end of life care: A conceptual Framework. Manuscript under review.

7 Luta X, Panczak R, Maessen M, Egger M, Goodman DC, Zwahlen M, Stuck AE, Clough-Gorr KM. Dying among older adults in Switzerland: who dies in hospital, who dies in a nursing home? BMC Palliative Care 2016;15(1):83.

8 Panczak R, Luta X, Maessen M, Stuck AE, Berlin C, Schmidlin K, Reich O, von Wyl V, Goodman DC, Egger M, Zwahlen M, CloughCorr KM. Regional Variation of Cost of Care in the Last 12 Months of Life in Switzerland: Small-Area Analysis Using Insurance Claims Data. Med Care 2016. doi: 10.1097/mlr.0000000000000634.

9 Panczak R, Luta X, Maessen M, Stuck AE, Berlin C, Schmidlin K, Reich O, von Wyl V, Goodman DC, Egger M, Clough-Gorr KM, Zwah len M. Death at no cost? Persons with no health insurance claims in the last year of life in Switzerland. Manuscript under review.
CH-3012 Bern

marcel.zwahlen[at]ispm.

unibe.ch
Korrespondenz:

Marcel Zwahlen

Finkenhubelweg 11 